\title{
Sacroiliac Joint Fusion: Fusion Rates and Clinical Improvement Using Minimally Invasive Approach and Intraoperative Navigation and Robotic Guidance
}

\author{
Mehul Sarkar ${ }^{1}$, Joseph Maalouly ${ }^{2}$, Sameer Ruparel ${ }^{3}$, John Choi ${ }^{2}$ \\ ${ }^{1}$ Department of Orthopaedics Spine Surgery, Dr Vasantrao Pawar Medical College and Hospital, Nashik, India \\ ${ }^{2}$ Spine Ortho Clinic, The Bays Hospital, Mornington, VIC, Australia \\ ${ }^{3}$ Department of Orthopaedic Spine Surgery, Global Hospital, Mumbai, India
}

Study Design: This study adopted a retrospective study design.

Purpose: This study was designed to describe the fusion rate and technique and patient subjective improvement after sacroiliac (SI) joint fusion using a minimally invasive surgical (MIS) approach.

Overview of Literature: The SI joint can mimic radicular or discogenic pain localized to the lower back, gluteal region, or sacral region, posing a challenge in the diagnosis and treatment. This study determines the radiological fusion rate and patient reported subjective clinical outcomes of SI joint fusion using an MIS approach, comparing the use of the Rialto SI joint fusion system (Medtronic, Minneapolis, MN, USA) with the help of the Stealth Navigation System with the use of ExcelsiusGPS Robotic Navigation Platform (Globus Medical Inc., Audubon, PA, USA) using SI-LOK screws (Globus Medical Inc.).

Methods: In this retrospective study, 43 consecutive patients who underwent SI joint fusion between August 2017 and February 2020 were enrolled; $60 \mathrm{SI}$ joints were fused. The patients' fusion was documented on computed tomography or X-rays, and Visual Analog Scale (VAS) scores were used to determine patient subjective clinical outcomes.

Results: A total of 60 joints were fused, including 26 joints fused using robotic guidance and 34 joints fused using the Stealth Navigation System. Student $t$-test was used to compare the mean preoperative VAS score $(7.52 \pm 1.3)$ with the mean postoperative VAS score at the 12-month follow-up (1.43 \pm 1.22$)(p<0.05)$. The $S I$ joint fusion rate using this technique was $61 \%$ at 6 months, $96.4 \%$ at 12 months, and $100 \%$ at 18 months.

Conclusions: The use of navigation guidance or robotic assistance enables accurate percutaneous screw placement across the SI joint. The use of bone morphogenetic protein in the screw bore hastens fusion across the joint, improving patient-perceived pain.

Keywords: Sacroiliac joint fusion; Fusion rates; Minimally invasive surgical procedures; Navigation; Robotic surgical procedures

\footnotetext{
Received Feb 25, 2021; Revised Oct 22, 2021; Accepted Nov 8, 2021

Corresponding author: Mehul Sarkar

Department of Orthopedics Spine Surgery, Dr Vasantrao Pawar Medical College and Hospital, Vasantdada Nagar, Adgaon, Nashik-422003, Maharashtra, India

Tel: +91-0253-2303247, Fax: +91-0253-2220500 (extension 1254), E-mail: orthopedic@drvasantraopawarmedicalcollege.com

Co-corresponding author: John Choi

Spine Ortho Clinic, Suite 10, First Floor, The Bays Hospital, Vale Street, Mornington, VIC 3931, Australia

Tel: +61-3-5970-5377, Fax: +61-3-5947-5817, E-mail: john.choi@spineortho.com.au 


\section{Introduction}

The sacroiliac (SI) joint can mimic radicular or discogenic pain localized to the lower back, gluteal region, or sacral region, posing a challenge in the diagnosis and treatment [1]. In a large retrospective study by Bernard and Kirkaldy-Willis [2] in 1987, lower back pain was reported in $22.5 \%$ of 1,293 patients with SI dysfunction. Other authors have described similar findings, reporting symptomatic SI joint dysfunction prevalence rate of $15 \%-30 \%$ in patients with low back pain [3]. Additionally, altered biomechanics of the lower back, secondary to osteoarthritis, inflammatory arthritis, or trauma, causes overloading of the SI joint leading to hypermobility or aberrant joint mechanics at that joint [4]. Approximately $20 \%$ of women have peripartum lower back pain; the SI joint is the source of pain in $75 \%$ of these patients. The combination of hormonal, biomechanical, traumatic, and degenerative factors causes SI dysfunction in postpartum women [5]. Liliang et al. [6] and Ha et al. [7] have reported that approximately $40 \%$ of lumbosacral fusions can show radiographic degeneration of the SI joint 5 years postoperatively.

The management options for patients with SI joint pain have mainly focused on physical therapy and pain management methods involving medication, computed tomography (CT)-guided SI joint injections, and local radiofrequency ablation. For patients with chronic SI joint pain unamenable to conservative therapy, traditional open SI joint fusion is an option but is plagued with large incisions, autologous bone graft-related morbidity, long hospital stay, and non-weight bearing, which increases patient morbidity $[8,9]$. To overcome the morbidities related to traditional open SI joint fusion surgery, minimally invasive surgical (MIS) approaches are being explored with percutaneous placement of implants with good clinical results [10]. Some authors have reported significant improvements in symptoms after minimally invasive SI fusion in carefully selected patients with postpartum SI joint dysfunction [5]. Other authors have used hollow modular anchorage screws for percutaneous SI joint fusion with significant improvements in clinical scores at the 24-month follow-up [4]. Using intraoperative fluoroscopy to aid implant placement has been described extensively; however, stereotactic intraoperative image guidance with real-time screw placement has been postulated to provide precise implant placement using MIS approaches. Darr et al. [11] have reported high satisfaction rates 3 years after the use of triangular titanium implants inserted using an MIS technique. To the best of our knowledge, this is the first study that has documented the radiological fusion rate and patient-perceived clinical improvement after SI joint fusion using an MIS approach compared with those after SI joint fusion using the Rialto SI joint fusion system with the help of the Stealth Navigation System and ExcelsiusGPS Robotic Navigation Platform with hydroxyapatite-coated SI-LOK screws.

\section{Materials and Methods}

\section{Study design}

A retrospective review of 43 consecutive patients who underwent SI joint fusion from August 2017 to February 2020 was conducted. A total of 60 SI joints were fused using the Rialto SI joint fusion system (Medtronic, Minneapolis, MN, USA) with the help of intraoperative three-dimensional (3D) CT acquisition (O-Arm System; Medtronic, Dublin, Ireland), Stealth Navigation System (Medtronic), and ExcelsiusGPS Robotic Navigation Platform (Globus Medical Inc., Audubon, PA, USA). The study included all patients over 30 years of age diagnosed with SI joint dysfunction. All patients underwent comprehensive evaluation of history of symptoms and clinical tests, including provocation test for SI joint dysfunction. Moreover, radiological evidence of SI joint dysfunction was examined. All other conditions causing similar symptoms were ruled out before arriving on the diagnosis of SI joint dysfunction. All patients were initially treated using a conservative management protocol using physiotherapy, anti-inflammatory medications, and SI joint block. Patients who had temporary relief after SI block were offered surgical fusion. Patients with a history of SI joint fractures, tumors, or infections were excluded from the study. Patient demographics (including age, sex, and smoking status), preoperative Visual Analog Scale (VAS) scores, unilateral/bilateral joint fusion, and previous anteriorposterior fixation were noted in all cases. Follow-up was planned 4 weeks, 3 months, 6 months, and 12 months after surgical fusion. Erect radiographs were taken immediately, 3 months, and 1 year postoperatively. CT was performed at the 6-month follow-up in patients who did not show clinical improvement 3 months after surgical fusion. Radiographic evidence of interval fusion across the implants and/or obliteration of joint space around the 
SI joint was considered satisfactory fusion. Fusion was assessed by a clinical fellow who was not associated with the treatment of these patients. VAS scores were collected 12 months after surgical fusion. The patients were asked about recommending this surgery to other patients with similar complaints, and responses were noted 12 months after the intervention.

The patient cohort was informed of the study design and provided informed consent for participation in the study, and the approval from the Institutional Review Board of Ramsay Healthcare NSW/VIC Human Research Ethics Committee was obtained (IRB approval code: JM03152).

\section{Surgical technique}

The patients were placed under general anesthesia and positioned prone on a Wilson frame. Sterile draping was performed to expose both the buttocks and lower back. A 5-mm longitudinal stab incision was made over the posterior superior iliac spine for placing the navigation reference frame to provide an imaging reference for the Stealth Navigation System (Medtronic) or ExcelsiusGPS Robotic Navigation Platform (Globus Medical Inc.). An intraoperative CT scan was obtained using the O-Arm 2 system (Breakaway Imaging, Littleton, MA, USA). The CT scan was integrated to the Stealth Navigation System (Medtronic) and the ExcelsiusGPS Robotic Navigation Platform (Globus Medical Inc.). Using the navigation instrument, the entry points to accommodate two or three screws in different planes were marked on the skin after

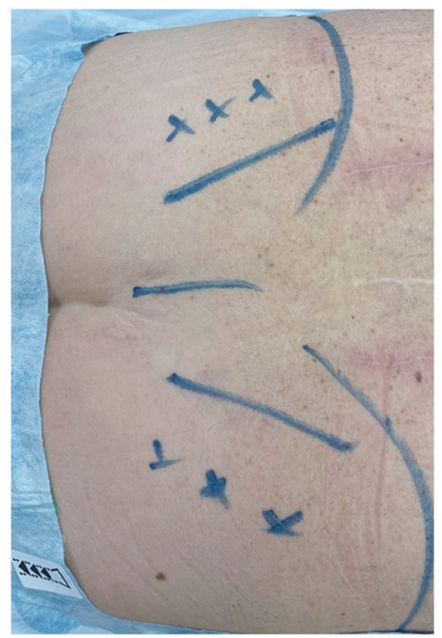

Fig. 1. Intraoperative markings of iliac crest, screw entry points, posterior superior iliac spine, and midline. confirming trajectory on axial, sagittal, and coronal images at those levels (Fig. 1). A small $1.5-\mathrm{cm}$ incision was made over each marked skin, which was carried deep to the bone level. Using a navigated cannula, a guide pin was placed as perpendicular to the synovial SI joint as possible. Then, a navigated high-speed drill was passed while keeping the trajectory using the navigation interface. The most common pattern used was one screw within each of S1 and S2. The length and diameter of each implant were estimated based on navigated projections. In robotic-guided cases, screws were placed using preplanned trajectories on the CT scan navigation interface of the robot. Convenient direct placement of screws was possible through the incision after drilling and tapping through the rigid robot arm. Fixation was provided using cannulated screws (Rialto SI fusion system v/s SI-LOK SI joint fixation system; Globus Medical Inc.) filled with allograft bone and bone morphogenetic protein (BMP) (INFUSE rhBMP2). All screws were navigated and placed. One-third of the 1.8mg INFUSE was used for each screw. All incisions were closed in standard fashion. In the postoperative period, the patients were allowed immediate weight bearing and mobilization as tolerated.

\section{Results}

A total of 43 patients underwent 60 SI joint fusions. The cohort comprised 27 females and 15 males with a mean age of 66 \pm 14 years (range, 33-88 years). All patients, except for one, were nonactive smokers. Among the 43 patients, 26 underwent unilateral SI joint fusion and 17 underwent primary bilateral joint fusion. Sixty joints were fused, among which 26 were fused using robotic guidance and 34 were fused using the Stealth Navigation System (Medtronic). Twenty-eight patients were diagnosed with primary SI joint dysfunction. Two patients with primary SI joint dysfunction had undergone trial with spinal stimulators without any effect. Twelve patients had previous lumbosacral fusion, and two patients had a floating lumbar fusion with the fusion construct ending at L5 with a functional L5/S1 motion segment. The minimum followup duration was 12 months. The mean follow-up duration of the patients in this study was 13.32 months.

\section{Functional outcome}

Student $t$-test was used to compare the mean preoperative 
VAS score (7.52 \pm 1.3 ) (range, 4-10) with the mean postoperative VAS score at the 12 -month follow-up $(1.43 \pm 1.22)$ $(p<0.05)$. Forty patients reported significant improvement in pain scores at the final follow-up. Statistically significant improvements in the VAS scores were observed $(p<0.05)$ (Table 1$)$. Three patients reported no change in their VAS scores compared with their preoperative pain scores. Moreover, Student t-test was used to evaluate postoperative VAS scores of patients who underwent fusion using ExcelsiusGPS Robotic Navigation (Globus Medical Inc.) and those who underwent fusion using the Stealth Navigation System (Medtronic) with a $p$-value of more than 0.05 , indicating nonsignificant difference between

Table 1. Comparison of VAS scores preoperative and postoperative with the respective $p$-value

\begin{tabular}{ll} 
VAS score & Value \\
\hline Preoperative & $7.52 \pm 1.3$ \\
Postoperative at final follow-up & $1.43 \pm 1.22$ \\
$p$-value & $<0.05$ \\
\hline
\end{tabular}

Values are presented as mean \pm standard deviation, unless otherwise stated. VAS, Visual Analog Scale.

Table 2. Robotic (SI-LOK) versus stealth navigated (RIALTO) screws

\begin{tabular}{lccc} 
Perioperative details & $\begin{array}{c}\text { No. of } \\
\text { patients }\end{array}$ & $\begin{array}{c}\text { Joints } \\
\text { fused }\end{array}$ & $\begin{array}{c}\text { Mean postoperative } \\
\text { VAS score }\end{array}$ \\
\hline Robotic fusion & 19 & 26 & $1.51 \pm 1.2$ \\
Stealth navigated fusion & 24 & 34 & $1.63 \pm 1.58$ \\
\hline Total & 43 & 60 & $>0.05$ \\
\hline$p$-value & & & \\
\hline
\end{tabular}

Values are presented as number or mean \pm standard deviation, unless otherwise stated.

VAS, Visual Analog Scale. the two groups (Table 2).

\section{Fusion}

Among the 43 patients, 11 (25.6\%) achieved stable fusion across the SI joint and obliteration of the joint space through and around the implants at the 3-month followup. One patient had evidence of fracture at the midpoint of the S2 screw, which was monitored over the next 3 months and healed completely at the 6-month follow-up with fusion across the SI joint. The remaining 32 patients underwent CT 6 months after fusion for evaluation. Among them, 25 achieved radiological fusion on CT scan. Twenty-five patients (58.1\%) had stable fusion across the SI joint with interval fusion and obliteration of the joint space at the end of the 6-month follow-up period. One patient with interval fusion at the 6-month follow-up had to undergo radiation therapy for non-Hodgkin's lymphoma. There was no evidence of any loosening or halo around the screws; however, obliteration of the joint space was not observed on regular plain radiographs at the 12-month follow-up in this case. Among the 43 patients, 41 (95\%) achieved stable fusion across the SI joint and obliteration of the joint space around the implants at the final followup (Figs. 2, 3). Two patients had evidence of halo around the S2 screw detected 6 months after fusion, which was replaced with a larger screw in one instance and augmented with a third screw in the other. Both patients were followed up for 18 months and had radiological fusion CT scans. Among the 43 patients, two-screw construct was used in 41 patients and three-screw construct was used in two patients. Thus, the fusion rate of the SI joint using our technique was $61.7 \%(36 / 60) 6$ months after fusion, $96.4 \%$ (58/60) 12 months after fusion, and 100\% (60/60)
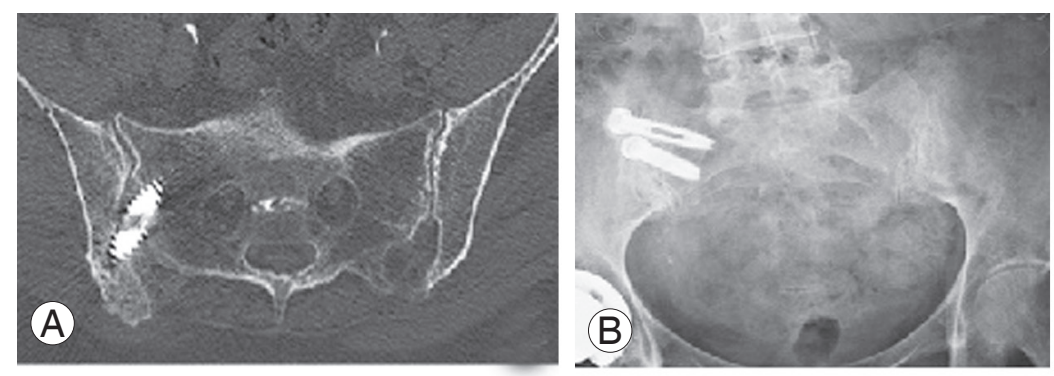

Fig. 2. Patient at 12 months post sacroiliac joint (SIJ) fusion using 2 SI-LOK screws (Globus Medical Inc., Audubon, PA, USA). (A) Axial computed tomography scan of SIJ. (B) Anterior-posterior X-ray of SIJ. (C) Lateral right SIJ X-ray.

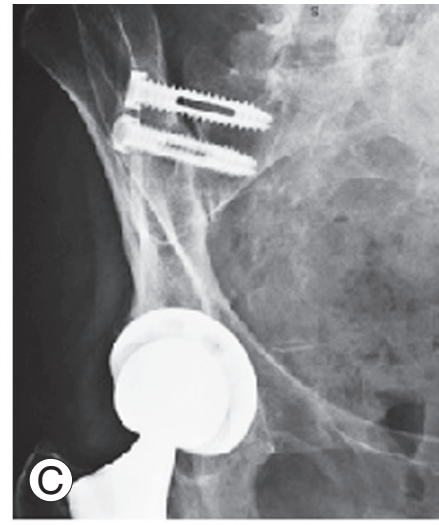



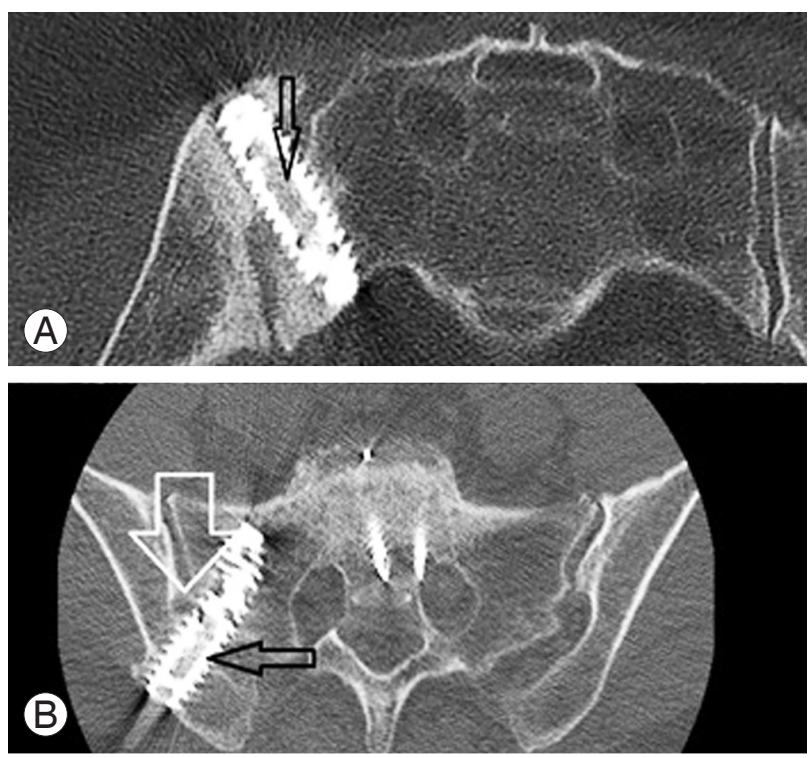

Fig. 3. (A, B) Interval fusion across the sacroiliac screw was seen on a computed tomography scan at 6 months. (A) The arrow points towards consolidated bone graft material across the screw bore indicating fusion. (B) The larger arrow points towards bony bridging around the screw and the smaller arrow pointing interval fusion across the sacroiliac joint.

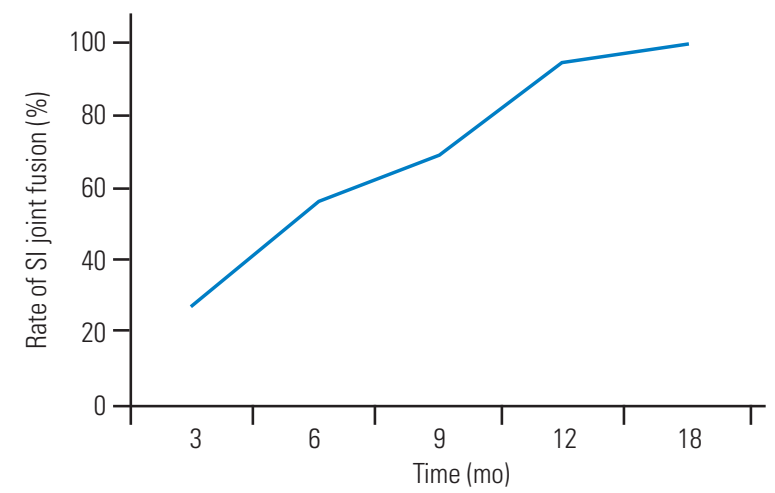

Fig. 4. Rate of sacroiliac (SI) joint fusion.

18 months after fusion (Fig. 4).

\section{Questionnaire}

Thirty-eight patients reported satisfactory results recommending the surgery to other patients with similar symptoms and diagnoses. The remaining five patients were unsure of recommending this procedure to others. The patient who underwent radiation therapy for nonHodgkin's lymphoma was unsatisfied with his outcome.

\section{Discussion}

SI joint pathology can have variable presentations.
Goldthwait and Osgood [12] first reported that the SI joint can be a source of unexplained buttock, low back, and leg pain. Such a vague presentation requires a thorough clinical assessment to correctly identify the pain generators. Positive provocation maneuvers, such as flexion, abduction, and external rotation, Gaenslen test, thigh thrust along with a marked reduction of symptoms on image-guided SI joint injection, can be considered reliable markers for diagnosing SI joint-related pain $[13,14]$.

Different fusion techniques have been described for SI joint arthrodesis; however, none have been widely acceptable. The classical technique described by Smith-Peterson $[8,9]$ used a large open approach with allogenic graft insertion, which was improved by Verrall [15] and Pitkin [16]. Such open techniques modified even with the use of modern fixation devices are associated with increased blood loss and surgical time and mixed results $[8,9,17,18]$. In contrast, Rudolf [19] has studied a cohort of 50 patients with percutaneous fusion using the iFuse triangular titanium implant system for SI arthritis, with favorable clinical outcomes in $82 \%$ patients, who reported significant clinical improvements at all time points. Another study has reported long-lasting clinical improvements using minimally invasive triangular titanium SI joint fusion implants in a 5-year follow-up [20]. Similarly, studies by Khurana et al. [21] and Mason et al. [22] have reported encouraging outcomes using hollow modular anchorage screws for SI joint fusion using a minimally invasive approach. Minimally invasive techniques show promising early and midterm results.

MIS SI fusions are not without shortcomings. Zaidi et al. [23] reviewed several studies, including 299 MIS SI fusions with a mean follow-up period of 21 months. They have reported radiographically confirmed fusion rates from $18 \%$ to $100 \%$; however, this review noted that over $80 \%$ of the studies did not include image-confirmed anatomic fusions as part of the outcome assessment. Duhon et al. [24] have reported bridging bone fusion across the SI joint in $87 \%$ of patients at the 12 -month follow-up using a percutaneous SI arthrodesis using triangular implants. Fusion rates for MIS SI fusion from other studies with dedicated radiographic imaging have been reported between $87 \%$ and $97 \%$ [24,25]. Beck et al. [26] have reported a fusion rate of $96.9 \%$ in 20 patients with a mean followup duration of 27 months using INFUSE rhBMP2 along with single-threaded titanium cages. This study reported a progressive fusion trend over 12 months of follow-up. 
The authors believe that a larger bore and fenestrations of the screws in the Rialto system (Medtronic) and hydroxyapatite coating with slotted screws in the SI-LOK system (Globus Medical Inc.) along with BMP might allow rapid fusion across the SI joint. Seventy percent of patients showed evidence of interval fusion and fusion across the SI joint. Forty-one patients (58 SI joints) were completely fused 12 months after surgical fusion, which is comparable to those reported in other studies in the literature. This study reported $100 \%$ fusion at the 18 -month followup, which is faster than others reported in the literature.

A few studies have reviewed the complications of MIS SI fusion. Reoperation rates of $0 \%-17 \%$ have been reported in the literature [27]. This study reported a reoperation rate of $4.6 \%$ ( $2 / 43$ cases) due to loosening of the S2 screw. In one of these cases, the SI joints were deemed fused; however, an additional third screw was inserted to augment the fusion across the SI joints; in another patient, fearing nonunion across the joint, the loose screws were replaced with a large-bore rescue screw at the surgeon's discretion. A recent study by Schoell et al. [28] has reported a complication rate of $16.4 \%$ at the 6-month follow-up in MIS SI joint fusions; however, they included a new lumbar pathology, infection, and postoperative pain in the assessment. We did not encounter any complications of infection or novel lumbar pathology. Pain persisted in two patients who reported VAS scores of 5 and 6 , respectively, even at the 12-month follow-up. We believe that the persistence of pain in the two patients was due to multimodal pain generators and not specifically arising from SI joint dysfunction.

The MIS technique relies upon the stabilization of the joint without direct fusion with decortication and could delay loosening. There is no consensus regarding the number of implants needed to achieve fusion across the SI joint; the authors believe that a two-screw construct may be adequate in most cases. A single-screw implant may allow continued micro-motion across the joint, leading to delayed fusion or nonunion [29]. In contrast, a threescrew construct provides complete stability compared with any combination of the two-screw constructs as shown by finite element analysis [30].

Many authors have reported using fluoroscopic navigation for minimally invasive SI joint fusion $[24,27,31]$. Lee et al. [32] have reported a percutaneous technique for SI joint fusion using $\mathrm{O}$-arm multidimensional surgical imaging with navigation. We noted similar benefits in using a 3D navigation technique (Medtronic), allowing for more precise and safer implant placement. Regarding screw loosening in the two female patients in this study, the literature suggests a higher likelihood of this phenomenon to occur in this gender [33].

This study reported a significant clinical improvement and reduction in the postoperative VAS scores over the course of the follow-up. The final follow-up for the clinical VAS scores was performed 12 months after surgical fusion. The patient cohort had reported a mean postoperative VAS score of $1.43 \pm 1.22$, indicating a statistically significant improvement $(p<0.05)$ compared with the mean preoperative VAS score $(7.52 \pm 1.3)$.

This study has several limitations. This is a single-surgeon, single-center, retrospective study with a small sample size with a corresponding risk of bias. The absence of a control group and heterogeneous patient cohort is also a limitation of this study. Assessing radiological fusion on fluoroscopy is difficult; moreover, fusion was assessed by a relatively inexperienced clinical fellow, which might be a limitation of this study. Similarly, many demographic factors influence fusion, which were not evaluated in this study. These results may not be generalizable as this technique uses $\mathrm{O}$-arm, navigation, and robot, which may not be available in other centers.

\section{Conclusions}

We believe that our technique of navigation- and robotassisted percutaneous SI joint fusion using slotted threaded implants with BMP shows faster rates of fusion across the SI joint. Both implants show similar outcomes. The significant improvement in clinical outcomes in a short duration can be explained by minimal tissue trauma and accurate placement of implants made possible by applying image-guided navigation systems.

\section{Conflict of Interest}

No potential conflict of interest relevant to this article was reported.

\section{Author Contributions}

Mehul Sarkar: conception of work, acquisition, analysis and drafting; Joseph Maalouly: acquisition, analysis and interpretation of data; Sameer Ruparel: acquisition, analysis and drafting, critical revision; and John Choi: 
supervision, critical revision, final approval of the version, administrative support.

\section{References}

1. Sembrano JN, Polly DW Jr. How often is low back pain not coming from the back? Spine (Phila Pa 1976) 2009;34:E27-32.

2. Bernard TN Jr, Kirkaldy-Willis WH. Recognizing specific characteristics of nonspecific low back pain. Clin Orthop Relat Res 1987;(217):266-80.

3. Schwarzer AC, Aprill CN, Bogduk N. The sacroiliac joint in chronic low back pain. Spine (Phila Pa 1976) 1995;20:31-7.

4. Al-Khayer A, Hegarty J, Hahn D, Grevitt MP. Percutaneous sacroiliac joint arthrodesis: a novel technique. J Spinal Disord Tech 2008;21:359-63.

5. Capobianco R, Cher D; SIFI Study Group. Safety and effectiveness of minimally invasive sacroiliac joint fusion in women with persistent post-partum posterior pelvic girdle pain: 12-month outcomes from a prospective, multi-center trial. Springerplus 2015;4:570.

6. Liliang PC, Lu K, Liang CL, Tsai YD, Wang KW, Chen HJ. Sacroiliac joint pain after lumbar and lumbosacral fusion: findings using dual sacroiliac joint blocks. Pain Med 2011;12:565-70.

7. Ha KY, Lee JS, Kim KW. Degeneration of sacroiliac joint after instrumented lumbar or lumbosacral fusion: a prospective cohort study over five-year followup. Spine (Phila Pa 1976) 2008;33:1192-8.

8. Smith-Petersen MN. Arthrodesis of the sacroiliac joint: a new method of approach. J Bone Joint Surg 1921;3:400-5.

9. Buchowski JM, Kebaish KM, Sinkov V, Cohen DB, Sieber AN, Kostuik JP. Functional and radiographic outcome of sacroiliac arthrodesis for the disorders of the sacroiliac joint. Spine J 2005;5:520-9.

10. Rudolf L. MIS fusion of the SI joint: does prior lumbar spinal fusion affect patient outcomes? Open Orthop J 2013;7:163-8.

11. Darr E, Meyer SC, Whang PG, et al. Long-term prospective outcomes after minimally invasive transiliac sacroiliac joint fusion using triangular titanium implants. Med Devices (Auckl) 2018;11:113-21.

12. Goldthwait JE, Osgood RB. A consideration of the pelvic articulations from an anatomical, pathological and clinical standpoint. Boston Med Surg J
1905; 152:593-601.

13. Bernard TN, Cassidy JD. The sacroiliac joint syndrome: pathophysiology, diagnosis, and management. In: Frymoyer JW, editor. The adult spine: principles and practice. New York (NY): Raven Press; 1991. p. 2107-30.

14. Dreyfuss P, Michaelsen M, Pauza K, McLarty J, Bogduk N. The value of medical history and physical examination in diagnosing sacroiliac joint pain. Spine (Phila Pa 1976) 1996;21:2594-602.

15. Verrall PJ. A bone graft for sacro-iliac fixation. J Bone Joint Surg 1926;8:491-3.

16. Pitkin HC. Sacrarthrogenetic telalgia: 5. a plan for treatment. J Bone Joint Surg 1937;19:169-84.

17. Schutz U, Grob D. Poor outcome following bilateral sacroiliac joint fusion for degenerative sacroiliac joint syndrome. Acta Orthop Belg 2006;72:296-308.

18. Kim JT, Rudolf LM, Glaser JA. Outcome of percutaneous sacroiliac joint fixation with porous plasmacoated triangular titanium implants: an independent review. Open Orthop J 2013;7:51-6.

19. Rudolf L. Sacroiliac joint arthrodesis-MIS technique with titanium implants: report of the first 50 patients and outcomes. Open Orthop J 2012;6:495-502.

20. Rudolf L, Capobianco R. Five-year clinical and radiographic outcomes after minimally invasive sacroiliac joint fusion using triangular implants. Open Orthop J 2014;8:375-83.

21. Khurana A, Guha AR, Mohanty K, Ahuja S. Percutaneous fusion of the sacroiliac joint with hollow modular anchorage screws: clinical and radiological outcome. J Bone Joint Surg Br 2009;91:627-31.

22. Mason LW, Chopra I, Mohanty K. The percutaneous stabilisation of the sacroiliac joint with hollow modular anchorage screws: a prospective outcome study. Eur Spine J 2013;22:2325-31.

23. Zaidi HA, Montoure AJ, Dickman CA. Surgical and clinical efficacy of sacroiliac joint fusion: a systematic review of the literature. J Neurosurg Spine 2015;23:59-66.

24. Duhon BS, Bitan F, Lockstadt H, et al. Triangular titanium implants for minimally invasive sacroiliac joint fusion: 2-year follow-up from a prospective multicenter trial. Int J Spine Surg 2016;10:13.

25. Abbasi H, Hipp JA. The assessment of fusion following sacroiliac joint fusion surgery. Cureus 2017;9:e1787. 
26. Beck CE, Jacobson S, Thomasson E. A retrospective outcomes study of 20 sacroiliac joint fusion patients. Cureus 2015;7:e260.

27. Wise CL, Dall BE. Minimally invasive sacroiliac arthrodesis: outcomes of a new technique. J Spinal Disord Tech 2008;21:579-84.

28. Schoell K, Buser Z, Jakoi A, et al. Postoperative complications in patients undergoing minimally invasive sacroiliac fusion. Spine J 2016;16:1324-32.

29. Cleveland AW 3rd, Nhan DT, Akiyama M, Kleck CJ, Noshchenko A, Patel VV. Mini-open sacroiliac joint fusion with direct bone grafting and minimally invasive fixation using intraoperative navigation. J Spine Surg 2019;5:31-7.
30. Lindsey DP, Kiapour A, Yerby SA, Goel VK. Sacroiliac joint stability: finite element analysis of implant number, orientation, and superior implant length. World J Orthop 2018;9:14-23.

31. Polly DW, Swofford J, Whang PG, et al. Two-year outcomes from a randomized controlled trial of minimally invasive sacroiliac joint fusion vs. nonsurgical management for sacroiliac joint dysfunction. Int J Spine Surg 2016;10:28.

33. Lee DJ, Kim SB, Rosenthal P, Panchal RR, Kim KD. Stereotactic guidance for navigated percutaneous sacroiliac joint fusion. J Biomed Res 2016;30:162-7.

33. Joukar A, Kiapour A, Elgafy H, Erbulut DU, Agarwal AK, Goel VK. Biomechanics of the sacroiliac joint: surgical treatments. Int J Spine Surg 2020;14:355-67. 